STRUCTURAL BIOLOGY COMMUNICATIONS

ISSN 2053-230X

Received 22 June 2015

Accepted 2 September 2015

Edited by N. Sträter, University of Leipzig, Germany

₹ Present address: Department of Biochemistry, Max Planck Institute for Developmental Biology, Spemannstrasse 35, 72076 Tübingen, Germany.

Keywords: RNA; noncanonical base pair.

PDB reference: RNA 20-mer, 5c5w

Supporting information: this article has supporting information at journals.iucr.org/f

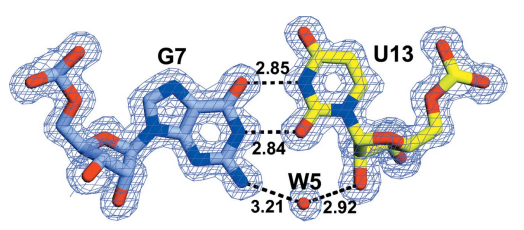

OPEN $\odot$ ACCESS

\section{$1.25 \AA$ resolution structure of an RNA 20-mer that binds to the TREX2 complex}

\author{
Eugene Valkov $¥$ and Murray Stewart*
}

MRC Laboratory of Molecular Biology, Francis Crick Avenue, Cambridge Biomedical Campus, Cambridge CB2 OQH, England. *Correspondence e-mail: ms@mrc-Imb.cam.ac.uk

The $1.25 \AA$ resolution $H 32: R$ crystal structure of a $20 \mathrm{nt}$ ribonucleotide that binds to the TREX-2 complex with high affinity shows a double-stranded RNA duplex arranged along a crystallographic $3_{1}$ axis in which the antiparallel chains overlap by 18 nucleotides and are related by a crystallographic twofold axis. The duplex shows $\mathrm{C}-\mathrm{A}, \mathrm{U}-\mathrm{U}$ and $\mathrm{C}-\mathrm{C}$ noncanonical base pairings together with canonical Watson-Crick A-U and $\mathrm{G}-\mathrm{C}$ pairs and a $\mathrm{G}-\mathrm{U}$ wobble.

\section{Introduction}

The yeast TREX2 complex functions to integrate the nuclear components of the gene-expression pathway with mRNA nuclear export as well as localizing actively expressing genes such as GAL1 to nuclear pores (NPCs). TREX2 is based on a Sac3 scaffold, to which Thp1, Sem1, Cdc31 and two chains of Sus1 bind. The complex can be divided into three regions, each of which has been characterized structurally and functionally: (i) the CID region, containing Cdc31, both Sus1 chains and Sac3 residues 727-805 (Jani et al., 2009), that interacts with nuclear basket components such as Nup1 and, in addition to facilitating mRNA export, functions to localize actively transcribing genes to NPCs (Jani et al., 2014), (ii) the N-terminal region of Sac3 (residues 1-140) that binds to the principal yeast mRNA nuclear export factor, Mex67-Mtr2 (Dimitrova et al., 2015), and (iii) the M-region, in which Thp1 and Sem1 bind to Sac3 residues 250-563 and which contains two juxtaposed winged-helix domains that bind RNA (Ellisdon et al., 2012). Previous work indicated a level of specificity in RNA binding by TREX2 in that polyuridine binds more strongly than other polyribonucleotides (Ellisdon et al., 2012), and studies on the human analogue of TREX2 that is based on GANP (the Sac3 homologue) also showed some specificity in binding to a subset of transcripts (Wickramasinghe et al., 2014). Here, we report the $1.25 \AA$ resolution crystal structure of a $20 \mathrm{nt}$ RNA fragment identified as having a higher affinity for TREX2 than polyuridine.

\section{Methods and materials}

An RNA oligonucleotide with the sequence ACCUGAGUUCAAUUCUAGCG was synthesized and HPLC-purified by Integrated DNA Technologies (Interleuvenlaan, Belgium) and dissolved at a concentration of $1.0 \mathrm{~m} M$ in $10 \mathrm{~m} M$ Tris- $\mathrm{HCl}$ $\mathrm{pH}$ 7.4. No further steps involving thermal denaturation and cooling were taken to promote duplex formation and additional magnesium chloride was not added to the sample. Crystallization conditions were screened using sparse-matrix 
Table 1

Crystallization conditions.

\begin{tabular}{|c|c|}
\hline Method & Vapour diffusion \\
\hline Plate type & Sitting-drop MRC 2-drop \\
\hline Temperature $(\mathrm{K})$ & 291 \\
\hline RNA concentration & $1.0 \mathrm{~m} M$ \\
\hline RNA solution & $10 \mathrm{~m} M$ Tris- $\mathrm{HCl}$ pH 7.4 \\
\hline Reservoir solution & $\begin{array}{l}2.0 M\left(\mathrm{NH}_{4}\right)_{2} \mathrm{SO}_{4}, 0.2 M \mathrm{MaCl}, 0.1 \mathrm{M} \text { sodium } \\
\text { cacodylate } \mathrm{pH} 6.5\end{array}$ \\
\hline Volume and ratio of drop & $200 \mathrm{nl}, 1: 1$ \\
\hline Volume of reservoir $(\mu \mathrm{l})^{1}$ & 80 \\
\hline
\end{tabular}

formulations at $18^{\circ} \mathrm{C}$ by sitting-drop vapour diffusion using $\sim 200 \mathrm{nl}$ drops. Crystals were observed in many conditions after several days. A variety of morphologies including needles, clusters and large individual crystals were observed in PEG-based (PEG 2K MME and PEG ranging from 600 to 8K) conditions and conditions where inorganic salts (ammonium sulfate, lithium sulfate, sodium chloride) were the main precipitant and over a range of different $\mathrm{pH}$ values (ranging from 5.5 to 10.5). No further optimization from initial screening was carried out. For X-ray data collection, crystals were supplemented with $15-30 \%(v / v)$ glycerol prior to vitrification in liquid nitrogen.

Diffraction data were collected on beamline I04-1 at the Diamond Light Source, Didcot, England. Surprisingly, there was no clear correlation between crystal appearance and diffraction quality. Although almost all of the crystals tested produced powder diffraction patterns, one crystal grown in 2.0 $M$ ammonium sulfate, $0.2 M$ sodium chloride, $0.1 \mathrm{M}$ sodium cacodylate $\mathrm{pH} 6.5$ (Table 1) diffracted to high resolution and data were collected using a $\varphi$ increment of $0.5^{\circ}$. These crystals diffracted to $1.25 \AA$ resolution, with unit-cell parameters $a=39.9, b=39.3, c=156.9 \AA, \alpha=\beta=90, \gamma=120^{\circ}$ (Table 2). Owing to the possibility of twinning and the presence of noncystallographic symmetry, it was not possible
Table 2

Crystallographic data-collection statistics.

Values in parentheses are for the outer shell.

\begin{tabular}{ll}
\hline Diffraction source & $\begin{array}{c}\text { Diamond Light Source, undulator } \\
\text { beamline I04-1 }\end{array}$ \\
Wavelength $(\AA)$ & 0.9200 \\
Detector & Pilatus $2 \mathrm{M}$ \\
Rotation range per image $\left(^{\circ}\right)$ & 0.5 \\
Total rotation range & 500 \\
Exposure time per image $(\mathrm{s})$ & 0.15 \\
Crystal-to-detector distance $(\mathrm{mm})$ & 175 \\
Data-collection temperature $(\mathrm{K})$ & 100 \\
Space group & $H 32: R$ \\
Unit-cell parameters $\left(\AA^{\circ},{ }^{\circ}\right)$ & $a=b=39.9, c=156.87$, \\
& $\alpha=\beta=90, \gamma=120$ \\
Resolution range $(\AA)$ & $52.29-1.25(1.28-1.25)$ \\
Unique reflections & 13567 \\
Total observations & 319227 \\
$\langle I / \sigma(I)\rangle$ & $21.5(1.6)$ \\
$R_{\text {p.i.m. }}$ & $0.021(0.449)$ \\
$R_{\text {merge }}$ & $0.100(1.383)$ \\
Mean half-set correlation & $1.000(0.620)$ \\
Completeness $(\%)$ & $98.6(89.8)$ \\
Multiplicity & 23.5 \\
Wilson $B$ factor $\left(\AA^{2}\right)$ & 10.5 \\
\hline
\end{tabular}

to distinguish between $H 3: R$ and $H 32: R$ symmetry at this stage. Data were processed and reduced using $X D S$ (Kabsch, 2010) and AIMLESS (Evans, 2011). Twinning tests for $H 3: R$ symmetry were equivocal using phenix.xtriage (Adams et al., 2010) and thus it was thought prudent to initially attempt molecular replacement using $H 3: R$ symmetry. Conventional molecular replacement using models comprising helices and duplexes was unsuccessful, and thus a set of shell scripts was developed that used fragments of high-quality RNA models from the PDB together with several ab initio models to derive a series of low-scoring solutions in Phaser (McCoy et al., 2007). All of the solutions obtained, irrespective of their scores, were subjected to 20 cycles of simulated annealing in Cartesian

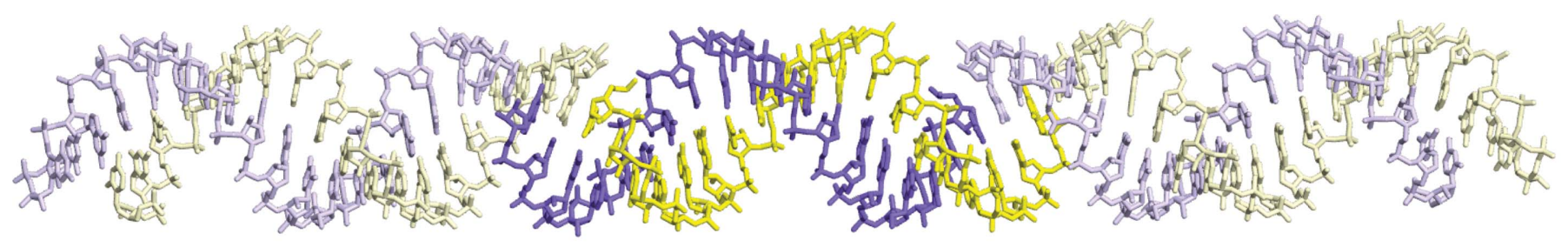

(a)

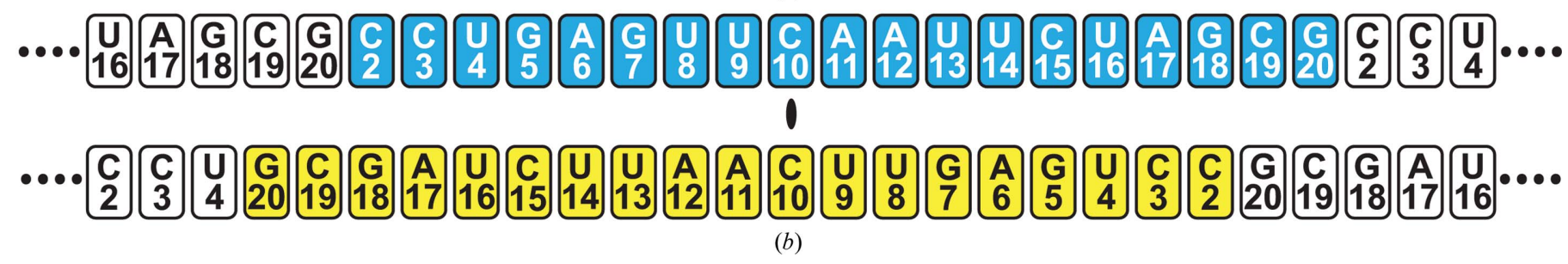

Figure 1

Generation of an RNA duplex by crystallographic symmetry. (a) In the $H 32: R$ crystal, the single chain in the asymmetric unit (blue) and its symmetry mate (yellow) related by a crystallographic twofold axis generate a double-stranded antiparallel RNA duplex (highlighted in more saturated colours) in which the two chains in the duplex overlap by 18 ribonucleotides, as illustrated schematically in $(b)$. C19 and G20 overlap with the next duplex to generate a continuous A-type RNA double helix along a crystallographic $3_{1}$ axis. In addition to the canonical base pairings C2-G18, A6-U14, U8-A12 and U9-A11, there is a classic wobble G7-U13 pair together with C3-A17, U4-U16 and C10-C10 noncanonical pairs. 
Table 3

Refinement statistics.

\begin{tabular}{ll}
\hline Non-H atoms & 462 \\
No. of water molecules & 97 \\
Bond-length deviation from ideal values $(\AA)$ & 0.0076 \\
Bond-angle deviation from ideal values $\left(^{\circ}\right)$ & 1.38 \\
All-atom clashscore & 1.43 \\
Reflections used for the working set in refinement & 12896 \\
Random reflections assigned for cross-validation $^{\circ}$ & 667 \\
$R_{\text {work }} / R_{\text {free }}(\%)$ & $17.9 / 18.8$ \\
\hline
\end{tabular}

space as implemented in phenix.refine (Afonine et al., 2012) and were then used as starting models for phenix.autobuild (Adams et al., 2010) in a fully automated protocol. Model improvement was monitored using both $R_{\text {free }}$ and correlation coefficients during successive rounds of density modification and reciprocal-space refinement. Several different models of highly truncated duplexes, comprising two and three WatsonCrick base pairs, converged to generate almost identical models with $R_{\text {free }}<40 \%$ and with clear features corresponding to bases and phosphate backbone in $2 m\left|F_{\mathrm{o}}\right|-D\left|F_{\mathrm{c}}\right|$ electrondensity maps together with features in the $m\left|F_{\mathrm{o}}\right|-D\left|F_{\mathrm{c}}\right|$ difference density maps that were not yet accounted for by the model. The density obtained was sufficiently good to enable purines and pyrimidines to be assigned and the ribonucleotide sequence to be fitted unequivocally. In the crystals, nucleotides 2-20 of the RNA formed an antiparallel helical duplex (Fig. 1) with its axis coincident with a crystallographic $3_{1}$ screw axis and one of the twofold axes relating the two chains coincident with the position of a crystallographic twofold axis of the $H 32: R$ unit cell. Consequently, this symmetry was adopted for further processing. The density corresponding to cytosine 10 that was related by this twofold was unusual and appeared to be the result of alternative conformations adopted by this nucleotide that also slightly influenced the adjacent nucleotides (Fig. 2). The influence of the twofold axis perpendicular to the $c$ axis on the distribution of diffraction intensities was very similar to that expected from $(h, k,-l)$ twinning. Iterative cycles of rebuilding using Coot (Emsley et al., 2010) and

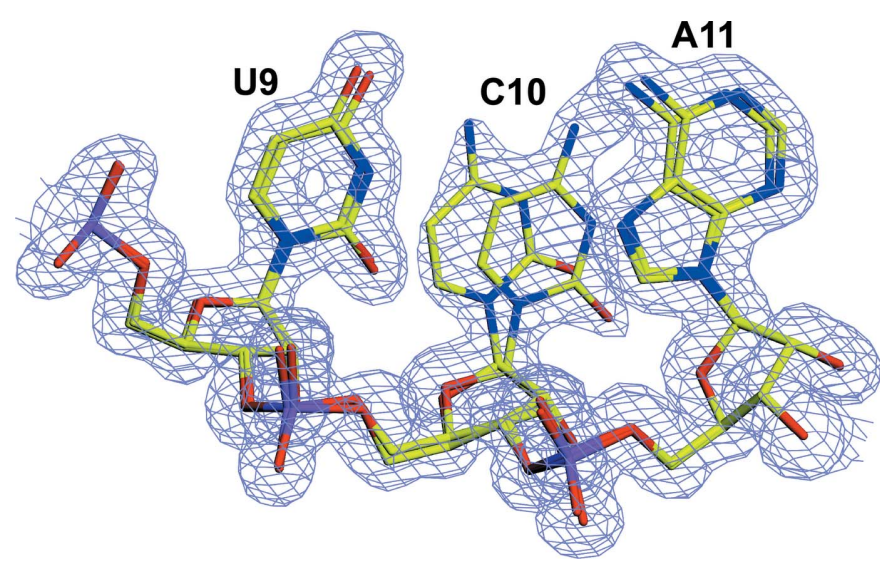

Figure 2

Alternate conformations adopted by ribonucleotides $9-11$. The occupancies of these alternative conformations refined as 0.52 and 0.48 , consistent with their being present in approximately equal proportions. The $2 m\left|F_{\mathrm{o}}\right|-D\left|F_{\mathrm{c}}\right|$ composite OMIT map is contoured at $1 \sigma$. refinement with phenix.refine (Afonine et al., 2012), introduction of anisotropic temperature factors and insertion of waters produced a model with an $R$ factor of $17.9 \%$ and an $R_{\text {free }}$ of $18.8 \%$ (Table 3 ). Some densities assigned to water probably represented ammonium ions that balanced the charge on the RNA phosphates but could not be identified

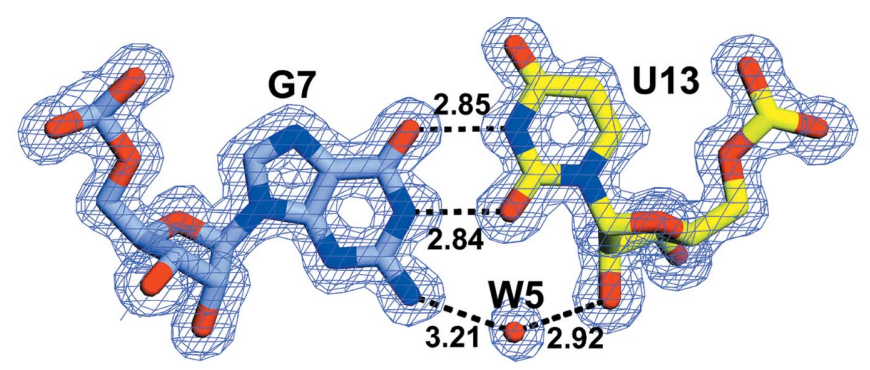

(a)

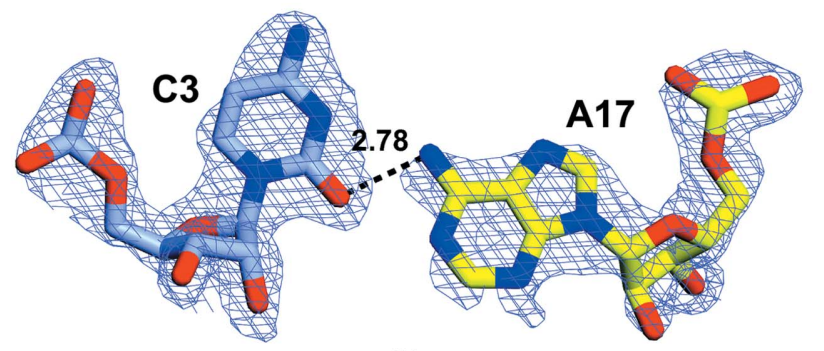

(b)

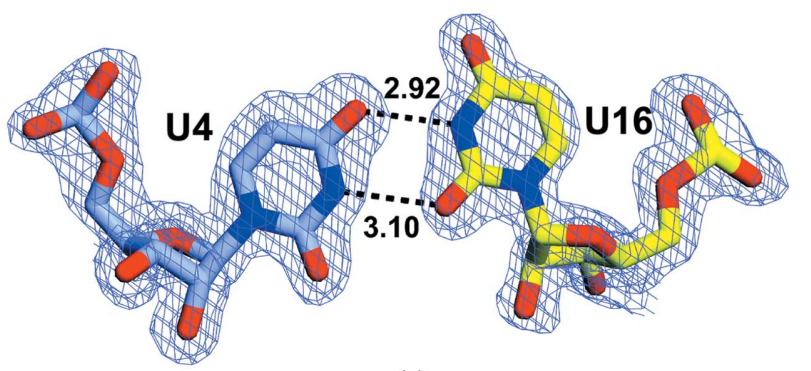

(c)

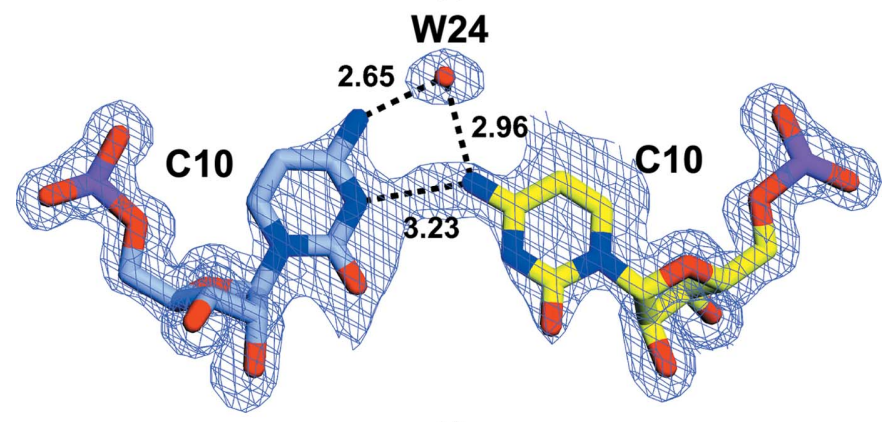

(d)

Figure 3

Noncanonical base pairings within the RNA duplex. (a) The G7-U13 wobble had the typical form supplemented by a water-bridged hydrogen bond involving guanine $\mathrm{N} 2$, water $\mathrm{W} 5$ and uridine ribose $\mathrm{O}^{\prime}$. (b) The C3A17 base pair was stabilized by a hydrogen bond between adenine N6 and cytosine $\mathrm{O} 4$, whereas the U4-U16 pairing $(c)$ was based on hydrogen bonds between $\mathrm{N} 3$ and $\mathrm{O} 4$ of each base. (d) Cytosine 10 adopted alternate conformations; one of each is shown. The alternate conformations formed $3.2 \AA$ amino-imino hydrogen bonds between N4 of one and $\mathrm{N} 3$ of the other, supplemented by putative hydrogen bonds between water W24 and N4 of both bases. The $2 m\left|F_{\mathrm{o}}\right|-D\left|F_{\mathrm{c}}\right|$ composite OMIT map is contoured at $1 \sigma$ and hydrogen-bond lengths are in $\AA$. 
unequivocally. The stereochemistry of the structure was assessed and validated with MolProbity (Chen et al., 2010), which indicated that there were no bond-length/angle outliers, that all ribose puckers were canonical and that the clashscore of 1.43 was in the top $98 \%$ of structures determined at this resolution.

\section{Results}

The C2-G18, G5-C15, A6-U14, U8-A12 and U9-A11 base pairings in the duplex were all of the canonical Watson-Crick form and that of G7-U13 (Fig. 3a) was of the canonical wobble form supplemented by a water-bridged hydrogen bond involving guanine $\mathrm{N} 2$, water 5 and uridine ribose $\mathrm{O} 2^{\prime}$. In the similar C3-A17 wobble pairing the adenine N6 formed a single hydrogen bond to $\mathrm{O} 4$ of $\mathrm{C} 3$ (Fig. $3 b$ ), which differs from the arrangement observed in the $2.5 \AA$ resolution structure of a 16-mer duplex (Pan et al., 1998), whereas the U4-U16 pairing (Fig. 3c) was based on hydrogen bonds between N3 and $\mathrm{O} 4$ of each base. Cytosine 10 adopted alternate conformations, but both formed $3.2 \AA$ amino-imino hydrogen bonds between N4 and N3 (Fig. 3d) supplemented by hydrogen bonds between water 24 and N4 of both bases. A similar amino-imino hydrogen bonding was observed in the Hepatitis $C$ virus internal ribosome entry site eIF3-binding site and thymidylate synthase mRNA, where multiple conformations of this base were also observed (Collier et al., 2002; Tavares et $a l ., 2009)$. There was no clear electron density for adenine 1 , which appeared to be disordered owing to its being displaced to facilitate the canonical $\mathrm{C}-\mathrm{G}$ pairings between bases 19 and 20 in consecutive duplexes along the crystallographic $3_{1}$ axis. Consistent with this interpretation, the electron density for the ribose of cytosine 2 was also weak.

Although the RNA formed a duplex in the crystals, it appeared to be monomeric in solution and probably formed a hairpin using base pairing analogous to that observed in the duplex. The formation of duplexes from hairpins is favoured at the high ionic strength and RNA concentrations employed for crystallization (Nakano et al., 2007).

\section{Acknowledgements}

This work was supported by the Medical Research Council (U105178939 to MS) and a Wellcome Trust Programme grant (MS). No conflicts of interest are declared. We are most grateful to our colleagues in Cambridge, especially Andrew Leslie, Phil Evans, Shintaro Aibara, Jimmy Gordon and Neil Marshall, for their many helpful comments, criticisms and suggestions.

\section{References}

Adams, P. D. et al. (2010). Acta Cryst. D66, 213-221.

Afonine, P. V., Grosse-Kunstleve, R. W., Echols, N., Headd, J. J., Moriarty, N. W., Mustyakimov, M., Terwilliger, T. C., Urzhumtsev, A., Zwart, P. H. \& Adams, P. D. (2012). Acta Cryst. D68, 352-367.

Chen, V. B., Arendall, W. B., Headd, J. J., Keedy, D. A., Immormino, R. M., Kapral, G. J., Murray, L. W., Richardson, J. S. \& Richardson, D. C. (2010). Acta Cryst. D66, 12-21.

Collier, A. J., Gallego, J., Klinck, R., Cole, P. T., Harris, S. J., Harrison, G. P., Aboul-Ela, F., Varani, G. \& Walker, S. (2002). Nature Struct. Biol. 9, 375-380.

Dimitrova, L., Valkov, E., Aibara, S., Flemming, D., McLaughlin, S. H., Hurt, E. \& Stewart, M. (2015). Structure, 23, 1246-1257.

Ellisdon, A. M., Dimitrova, L., Hurt, E. \& Stewart, M. (2012). Nature Struct. Mol. Biol. 19, 328-336.

Emsley, P., Lohkamp, B., Scott, W. G. \& Cowtan, K. (2010). Acta Cryst. D66, 486-501.

Evans, P. R. (2011). Acta Cryst. D67, 282-292.

Jani, D., Lutz, S., Marshall, N. J., Fischer, T., Köhler, A., Ellisdon, A. M., Hurt, E. \& Stewart, M. (2009). Mol. Cell, 33, 727-737.

Jani, D., Valkov, E. \& Stewart, M. (2014). Nucleic Acids Res. 42, 66866697.

Kabsch, W. (2010). Acta Cryst. D66, 133-144.

McCoy, A. J., Grosse-Kunstleve, R. W., Adams, P. D., Winn, M. D., Storoni, L. C. \& Read, R. J. (2007). J. Appl. Cryst. 40, 658-674.

Nakano, S., Kirihata, T., Fujii, S., Sakai, H., Kuwahara, M., Sawai, H. \& Sugimoto, N. (2007). Nucleic Acids Res. 35, 486-494.

Pan, B., Mitra, S. N. \& Sundaralingam, M. (1998). J. Mol. Biol. 283, 977-984.

Tavares, T. J., Beribisky, A. V. \& Johnson, P. E. (2009). RNA, 15, 911-922.

Wickramasinghe, V. O., Andrews, R., Ellis, P., Langford, C., Gurdon, J. B., Stewart, M., Venkitaraman, A. R. \& Laskey, R. A. (2014). Nucleic Acids Res. 42, 5059-5071. 